\title{
Alternativa energética sustentable mediante la utilización de aislantes térmicos de diferentes materiales en edificaciones con sistemas de aire acondicionado
}

\section{Sustainable energy alternative, by using termal insulators of different materials in buildings with air conditioning systems}

\author{
CASADOS-LÓPEZ, Edzel Jair†*, CASADOS-SÁNCHEZ, Alvaro, CRUZ-VICENCIO, Raúl у \\ HORST-SÁNCHEZ, Alvaro
}

\author{
ID $1^{\text {er }}$ Autor: Edzel Jair, Casados-López / ORC ID: 0000-0002-0601-9242 \\ ID $1^{\text {er }}$ Coautor: Alvaro, Casados-Sánchez / ORC ID: 0000-0002-3122-4571 \\ ID $2^{\text {do }}$ Coautor: Raúl, Cruz-Vicencio / ORC ID: 0000-0002-4668-0772 \\ ID $3^{\text {er }}$ Coautor: Alvaro, Horst-Sánchez / ORC ID: 0000-0002-2801-0943
}

Universidad Veracruzana, Facultad de Ingeniería Mecánica Eléctrica, Campus Poza Rica - Tuxpan

\section{Resumen}

Se propone una metodología para el cálculo de la carga de enfriamiento, y el consumo energético de los equipos de aire acondicionado en tres modelos a escala de edificaciones objeto de estudio, utilizando el método CLTD/SCL/CLF de la ASHRAE. La edificación en la que se utiliza el método mencionado son tres modelos a escala de edificaciones situados en la ciudad de Poza Rica estado de Veracruz, México. Este método se aplica con la finalidad de obtener la carga de enfriamiento lo más exacto posible y de esa manera evitar el sobredimensionamiento en los equipos de aire acondicionado, y al utilizar el aislante térmico, lograr una disminución en el consumo energético y de esa manera contribuir a la disminución de emisiones de $\mathrm{CO}_{2}$, al ahorro de energía y por ende al desarrollo sustentable. Se procede a calcular la carga de enfriamiento aplicando la metodología propuesta a tres casos: el modelo A, B y C. Se comparan los resultados para los tres modelos de prueba, objeto de este estudio. Se realizan mediciones de consumo energético para realizar el análisis de error del consumo real energético respecto al calculado utilizando el método. Por último, se cuantifica el ahorro energético, en los casos mencionados.

Carga de enfriamiento, Aislante térmico, Equipos de aire acondicionado

\begin{abstract}
A methodology is proposed for calculating the cooling load and the energy consumption of air conditioning equipment in three scale models of buildings under study, using the ASHRAE CLTD / SCL / CLF method. The building in which the mentioned method is used are three scale models of buildings located in the city of Poza Rica, state of Veracruz, Mexico. This method is applied in order to obtain the cooling load as exact as possible and thus avoid oversizing in air conditioning equipment, and by using thermal insulation, achieve a decrease in energy consumption and thus contribute to the reduction of $\mathrm{CO}_{2}$ emissions, to energy saving and therefore to sustainable development. The cooling load is calculated by applying the proposed methodology to three cases: model A, B and C. The results for the three test models, object of this study, are compared. Measurements of energy consumption are made to perform the error analysis of the actual energy consumption with respect to that calculated using the method. Finally, energy savings are quantified, in the cases mentioned.
\end{abstract}

Cooling load, Thermal insulator, Air Conditioning equipment

Citación: CASADOS-LÓPEZ, Edzel Jair, CASADOS-SÁNCHEZ, Alvaro, CRUZ-VICENCIO, Raúl y HORST-SÁNCHEZ, Alvaro. Alternativa energética sustentable mediante la utilización de aislantes térmicos de diferentes materiales en edificaciones con sistemas de aire acondicionado. Revista de Energías Renovables. 2020. 4-13: 1-8

*Correspondencia al Autor (correo electrónico: edcasados@uv.mx)

$\dagger$ Investigador contribuyendo como primer autor. 


\section{Introducción}

El acondicionamiento de aire ha sido uno de los más recientes y valorados servicios del hombre en su búsqueda por una existencia más confortable. El propósito primordial de un sistema de aire acondicionado ya sea calefacción o enfriamiento, es el de mantener las condiciones adecuadas, ya sea para proporcionar confort térmico a los ocupantes de una edificación o condiciones que sean requeridas por algunos productos y procesos dentro de la industria. Los sistemas de calefacción central fueron desarrollados en el siglo XIX mientras que el desarrollo de sistemas de enfriamiento con aplicación al confort llegó en el siglo XX. Desde entonces, los progresos en esta dirección han alcanzado grandes avances con significativo desarrollo en varias áreas de la ciencia y tecnología.

Los métodos de cálculo de carga pioneros ponen poca atención a los costos de operación y al aspecto relacionado con la sustentabilidad ambiental dando como resultado en el cálculo equipos sobredimensionados. Sin embargo, el aumento del precio de la energía, los materiales de construcción y las estructuras complejas en los edificios, así como todo lo concerniente a los recursos naturales y al medio ambiente han requerido un continuo refinamiento de los métodos de cálculo de carga. Los métodos de cálculo de carga en la actualidad se dirigen más hacia el dimensionamiento de sistemas de tamaño mínimo apropiado los cuales dan como resultado sistemas económicos con buen comportamiento energético y por lo tanto más acorde con la filosofía de un medio ambiente sustentable.

Por otro lado, el grado óptimo de protección térmica depende de criterios económicos y técnicos. Además, está determinada por consideraciones de las demandas de enfriamiento y de calefacción del edificio y de la factibilidad de la inversión necesaria para alcanzar el grado deseado de protección térmica del edificio. Como todos esos parámetros varían con respecto a las condiciones climáticas, la fluctuación de los factores de costo y la forma real en la cual los edificios son diseñados y construidos, la determinación de una protección térmica óptima está siempre sujeta a discusión.
Una alternativa para resolver este problema se presenta en este trabajo.

La energía consumida en edificios es usada para calefacción, refrigeración, iluminación y otras aplicaciones o equipos. El consumo depende de las condiciones climáticas, la arquitectura y las características constructivas del edificio, la ocupación y los parámetros operacionales, los diferentes sistemas de calefacción, aire acondicionado, y otros tipos de aparatos y equipos electromecánicos.

Es notable indicar que del total de clientes con que cuenta la Comisión Federal de Electricidad $88.13 \%$ pertenecen al sector doméstico, $0.43 \%$ al agrícola, $0.80 \%$ al industrial, $10.02 \%$ al comercial y $0.62 \%$ al de servicios. Como se observa el mayor número de clientes pertenece al sector doméstico lo que indica que un ahorro de energía en edificios y casas-habitación impacta de una manera significativa en los patrones de consumo de combustibles fósiles y por lo tanto en las emisiones de bióxido de carbono a la atmosfera.

Esas características de consumo tienen, aparte del efecto directo en los costos de operación de los edificios, un efecto significativo sobre el ambiente debido a la combustión de gas y combustibles líquidos fósiles (aceite, diesel, petróleo y combustóleo) en las centrales de generación de energía. Dado que en México los sistemas centrales de generación de energía eléctrica están basados en combustibles fósiles, reduciendo el consumo eléctrico en el sector de edificios se da un paso necesario hacia la implementación del protocolo de Kioto.

De esta manera, la necesidad de tomar medidas para ahorrar energía en el sector de edificios llega a ser importante con la finalidad de reducir el consumo de fuentes de energía convencionales, lo cual es un hecho que puede ofrecer mejoras económicas no solo a los propietarios de los edificios sino también a la economía nacional. Esta necesidad constituye también una obligación para México hacia la reducción de emisiones de $\mathrm{CO}_{2}$. En ese orden de ideas, una de las principales medidas hacia el ahorro de energía, aplicables a edificios nuevos o las ya existentes, es el mejoramiento de la protección térmica, como también mediante el uso de ventanas de alto rendimiento. 
La mejora en la protección térmica de edificios en su envolvente podría no solo conducir a reducir las pérdidas térmicas durante el periodo de invierno, sino también conducir a una reducción de la carga de enfriamiento en el verano, un problema que ha cobrado significativa importancia en la última década. Finalmente, el uso de sistemas de iluminación de bajo consumo energético, junto con la aplicación de dispositivos de sombra, constituyen nuevas acciones que pueden contribuir a la reducción del consumo energético para acondicionamiento de aire, a pesar del hecho de que ellos no están directamente relacionados con la protección térmica de edificios.

Por lo tanto, un método apropiado para el cálculo de carga de enfriamiento en una edificación junto con la elección de un aislante térmico apropiado constituye una manera efectiva para dimensionar adecuadamente un sistema de acondicionamiento de aire con la finalidad de proporcionar condiciones de confort minimizando el consumo energético y por lo tanto disminuyendo emisiones de bióxido de carbono hacia la atmosfera impactando esto en forma favorable a nuestro medio ambiente.

Por lo anterior en este trabajo se busca como objetivo proponer una metodología para el cálculo de la carga de enfriamiento en edificaciones objeto de estudio, en este caso son tres modelos a escala de edificaciones situados en la ciudad de Poza Rica estado de Veracruz, en México, con la finalidad de disminuir el consumo energético de un sistema de aire acondicionado reduciendo su capacidad y de esta manera lograr una disminución en las emisiones de $\mathrm{CO}_{2}$, contribuyendo al desarrollo sustentable.

\section{Características constructivas de los modelos a escala}

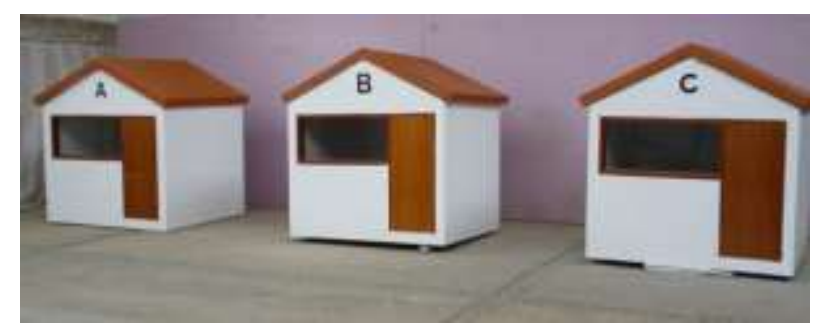

Figura 1 Modelos a escala A, B y C Fuente: Elaboración Propia

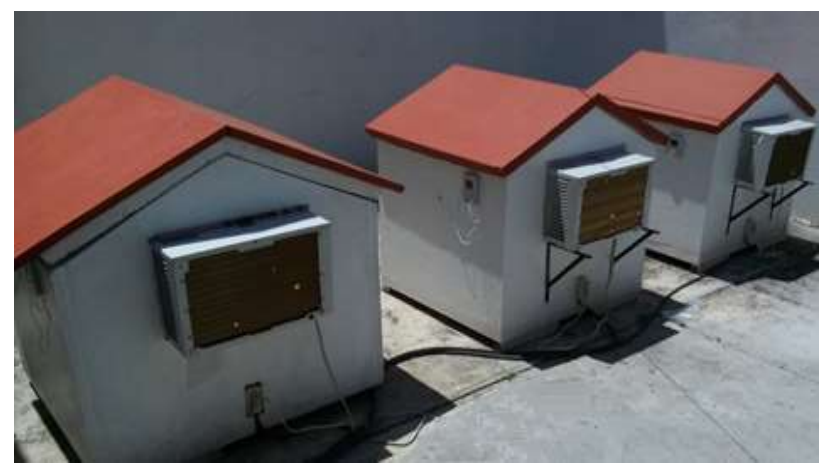

Figura 2 Vista Posterior de los modelos a escala A, B y C Fuente: Elaboración Propia

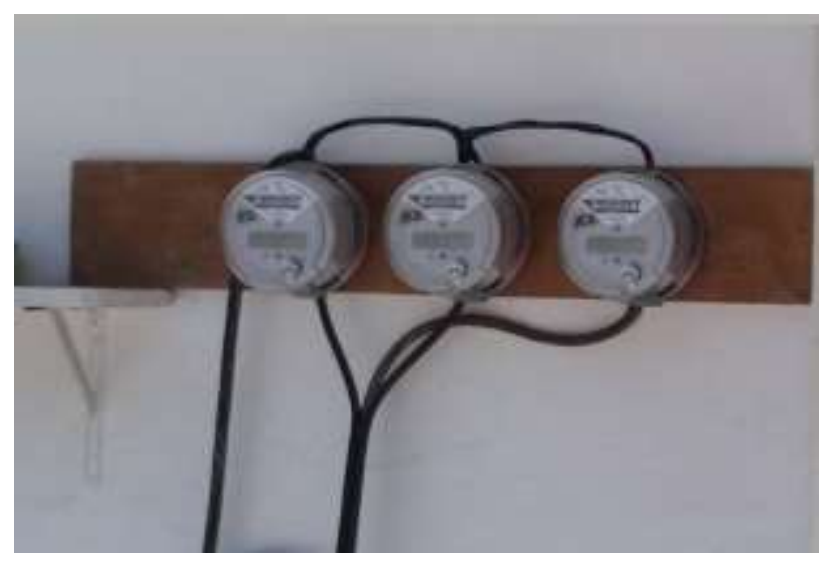

Figura 3 Medidores proporcionados por la CFE para la medición del consumo de energía

Fuente: Elaboración Propia

La construcción donde es aplicada la metodología de este trabajo, son tres modelos a escala de edificaciones, situados en la ciudad de Poza Rica de Hidalgo, Edo Veracruz en México, y sus características principales son para el Modelo A: Muros de ladrillo, Losa de concreto revocado de ambos lados, ventana de cristal sencillo claro y puerta de madera. Para el Modelo B: Muros de Block, Losa de Covintec recubierto con cemento -arena, Ventana de cristal sencillo claro y puerta de madera. Para el Modelo C: Construido de igual manera que le modelo B, pero a este se le colocó aislante térmico de 1 pulg. de espesor, dicho aislante es de poliestireno extruido para los muros y techo.

A continuación, se muestran los planos arquitectónicos de uno de los modelos ya que los tres son de las mismas dimensiones. 


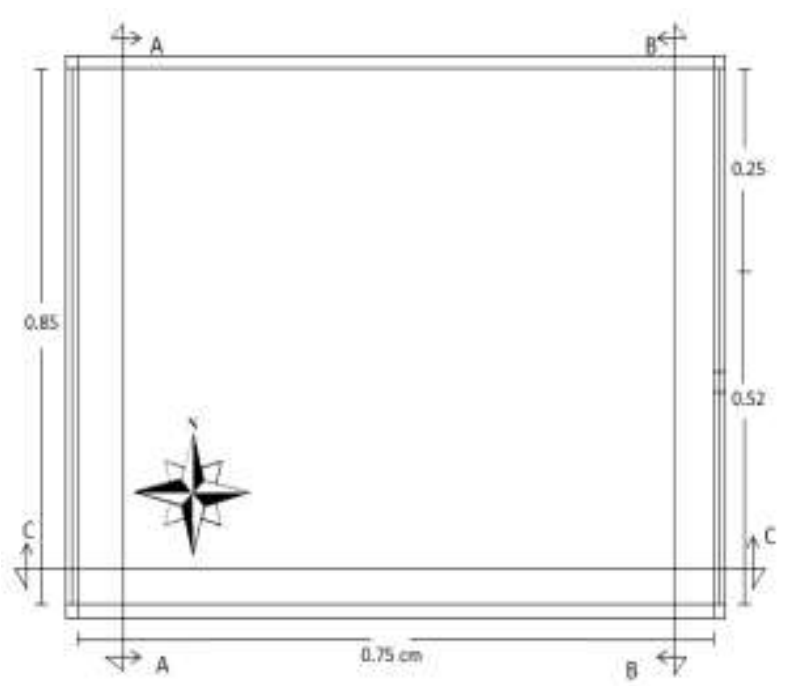

Figura 4 Planta de los modelos A, B y C Fuente: Elaboración Propia

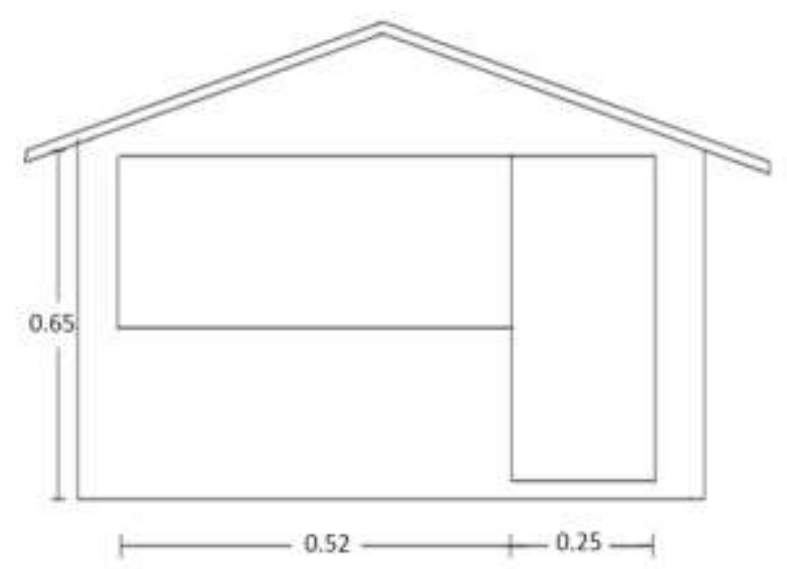

Figura 5 Corte B-B (metros) Fuente: Elaboración Propia

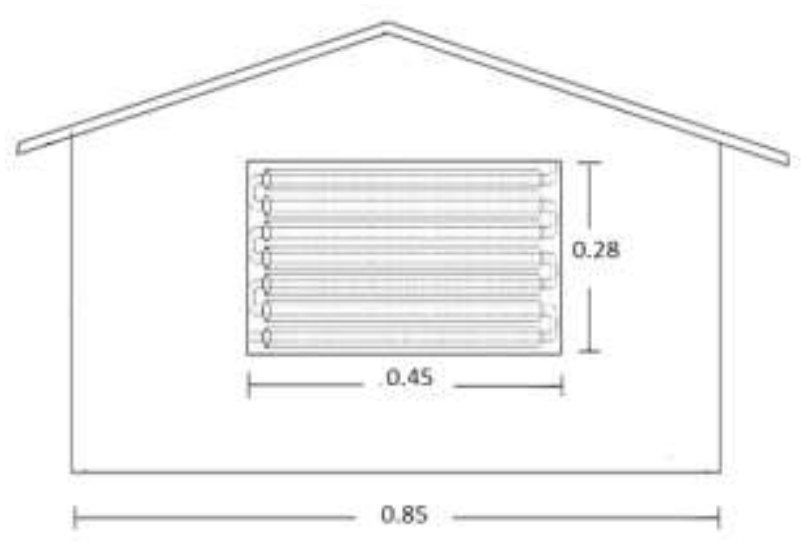

Figura 6 Corte A-A (metros) Fuente: Elaboración Propia

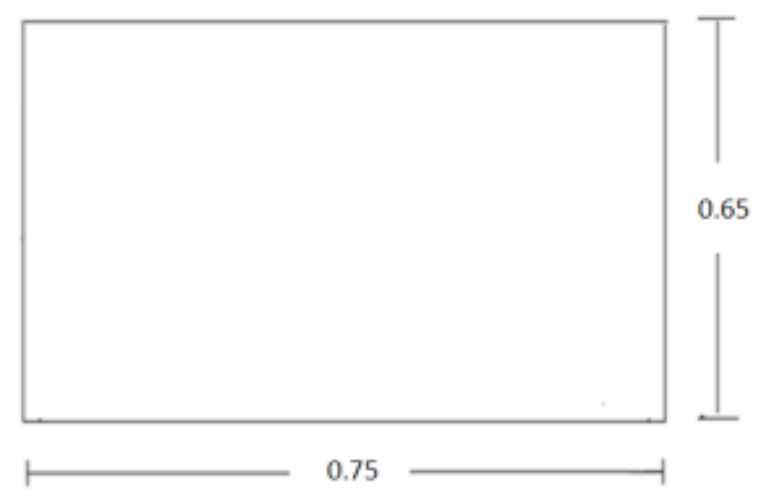

Figura 7 Corte $\mathrm{C}-\mathrm{C}$ (metros)

Fuente: Elaboración Propia

\section{Cálculo del Coeficiente Global de Transferencia de calor}

El cálculo de este coeficiente se lleva a cabo considerando cada uno de los materiales de que están compuestas las paredes y los techos. El aislante utilizado es de poliestireno extruido de 0.0254 metros de espesor con una conductividad térmica de 0.043 Watts $-\mathrm{m}{ }^{\circ} \mathrm{K}$. Los valores del coeficiente global te transferencia de calor resultante para cada elemento estructural y para la zona a climatizar, se presentan en la tabla 1 . Estos se derivan de los materiales y planos arquitectónicos que se utilizaron en el diseño y construcción de los modelos a escala de la edificación, que son los casos de estudio de este trabajo. En la tabla 1 se pueden observar que el comportamiento de los coeficientes globales de transferencia de calor depende de cada caso. En los casos donde no se tiene aislantes térmicos, los coeficientes son altos. En el caso en donde se aplica el aislante térmico se muestra una reducción en los valores de los coeficientes.

\begin{tabular}{|l|c|c|}
\hline Elementos estructurales & \multicolumn{1}{c|}{$\begin{array}{c}\text { Sin } \\
\text { aislamiento } \\
\text { térmico }\end{array}$} & $\begin{array}{c}\text { Con } \\
\text { aislamiento } \\
\text { térmico }\end{array}$ \\
\hline $\begin{array}{l}\text { Muro de ladrillo con } \\
\text { revoque de cemento- } \\
\text { arena en ambas caras }\end{array}$ & 4.3668 & \\
\hline $\begin{array}{l}\text { Muro de Block con } \\
\text { revoque de cemento- } \\
\text { arena en ambas caras }\end{array}$ & 4.3821 & 1.2210 \\
\hline $\begin{array}{l}\text { Losa de concreto armado } \\
\text { con revoque de cemento- } \\
\text { arena en ambas caras }\end{array}$ & 4.7722 & \\
\hline $\begin{array}{l}\text { Losa de covintec con } \\
\text { revoque de cemento- } \\
\text { arena en ambas caras }\end{array}$ & 1.2208 & \\
\hline Ventana de Cristal & 5.91 & \\
\hline Puerta de Madera & 4.40 & 0.6889 \\
\hline
\end{tabular}

Tabla 1 Coeficientes globales de transferencia de calor de los elementos de construcción (Watts $/ \mathrm{m}^{2}{ }^{\circ} \mathrm{K}$ )

Fuente: Elaboración Propia 


\section{Determinación de la demanda de enfriamiento}

Los cálculos de la demanda de enfriamiento se basaron en el método CLTD/SCL/CLF de la ASHRAE. Para ello fueron consideradas las siguientes condiciones: Latitud $20^{\circ} \mathrm{N}$, para el 21 de julio, temperatura interior de $24{ }^{\circ} \mathrm{C}$, temperatura exterior $38{ }^{\circ} \mathrm{C}$, porcentaje diario de temperatura $32{ }^{\circ} \mathrm{C}$, rango diario de temperatura $12{ }^{\circ} \mathrm{C}$, coeficiente de transmisión de calor por convección exterior $22.68 \mathrm{~W} / \mathrm{m}^{2}{ }^{\circ} \mathrm{K}$, coeficientes de transmisión de calor interior 7.48 $\mathrm{W} / \mathrm{m}^{2}-{ }^{\circ} \mathrm{K}$. Los valores CLTD corregidos se obtuvieron de acuerdo, a la siguiente ecuación:

$$
\begin{aligned}
& \mathrm{CLTD}_{\mathrm{Corr}}=(\mathrm{CLTD}+\mathrm{LM}) \mathrm{K}+\left(25.5-\mathrm{T}_{\mathrm{i}}\right)+ \\
& \left(\mathrm{T}_{\mathrm{OAV}}-29.4{ }^{\circ} \mathrm{C}\right)
\end{aligned}
$$

Dónde:

LM: Factor de corrección para latitud y mes

K: Factor de ajuste de color

$\mathrm{T}_{\mathrm{i}}$ y $\mathrm{T}_{\mathrm{OAv}}$ : Valores de diseño

$T_{i}$ : Temperatura interior

ToAv: Porcentaje diario de temperatura

Los gráficos 1 y 2 , muestran las tablas de los valores de CLTD corregidos para la losa y la pared Oeste. El gráfico 3, muestra la tabla de las demandas de enfriamiento para las horas de máxima carga, que en este caso es considerada de las 15 a $19 \mathrm{hrs}$. Sin aislante térmico, la demanda de enfriamiento es mayor, y en el otro caso, cuando se instala aislante térmico la carga de enfriamiento disminuye.

\begin{tabular}{|l|c|c|c|c|c|c|c|c|}
\hline HORAS & $1: 00$ & $2: 00$ & $3: 00$ & $4: 00$ & $5: 00$ & $6: 00$ & $7: 00$ & $8: 00$ \\
\hline Modelo $A$ & 12.4 & 9.4 & 8.4 & 6.4 & 5.4 & 4.4 & 5.4 & 8.4 \\
\hline Modelo $B$ & 6.4 & 5.4 & 4.4 & 3.4 & 2.4 & 2.4 & 3.4 & 7.4 \\
\hline Modelo $C$ & 14.4 & 11.4 & 9.4 & 7.4 & 6.4 & 4.4 & 3.4 & 3.4 \\
\hline \begin{tabular}{|l|c|c|c|c|c|c|c|c|}
\hline HORAS & $9: 00$ & $10: 00$ & $11: 00$ & $12: 00$ & $13: 00$ & $14: 00$ & $15: 00$ & $16: 00$ \\
\hline Modelo $A$ & 12.4 & 18.4 & 24.4 & 31.4 & 37.4 & 42.4 & 45.4 & 46.4 \\
\hline Modelo $B$ & 14.4 & 23.4 & 32.4 & 39.4 & 46.4 & 51.4 & 53.4 & 52.4 \\
\hline Modelo $C$ & 5.4 & 9.4 & 14.4 & 21.4 & 28.4 & 35.4 & 41.4 & 46.4 \\
\hline HORAS & $17: 00$ & $18: 00$ & $19: 00$ & $20: 00$ & $21: 00$ & $22: 00$ & $23: 00$ & $24: 00$ \\
\hline Modelo $A$ & 46.4 & 42.4 & 38.4 & 32.4 & 26.4 & 22.4 & 18.4 & 14.4 \\
\hline Modelo $B$ & 49.4 & 44.4 & 36.4 & 27.4 & 19.4 & 13.4 & 10.4 & 8.4 \\
\hline Modelo $C$ & 48.4 & 48.4 & 46.4 & 42.4 & 36.4 & 30.4 & 24.4 & 18.4 \\
\hline
\end{tabular}
\end{tabular}

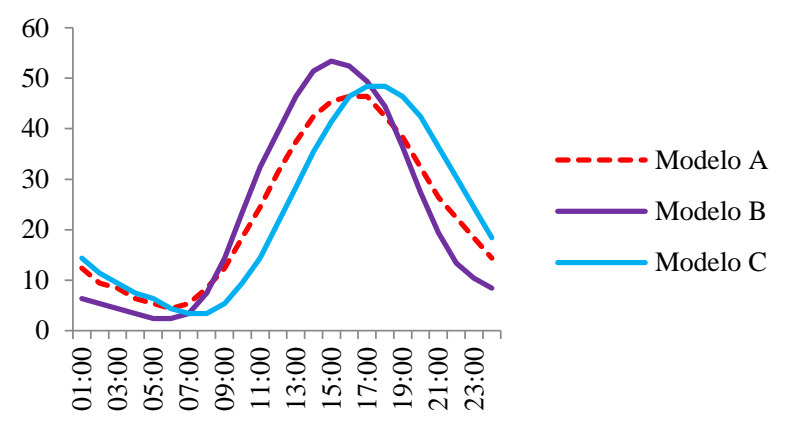

Gráfico 1 Valores CLTD corregidos para las losas de los tres casos, para las $24 \mathrm{hrs}$.

Fuente: Elaboración Propia

ISSN 2523-2881

ECORFAN® Todos los derechos reservados
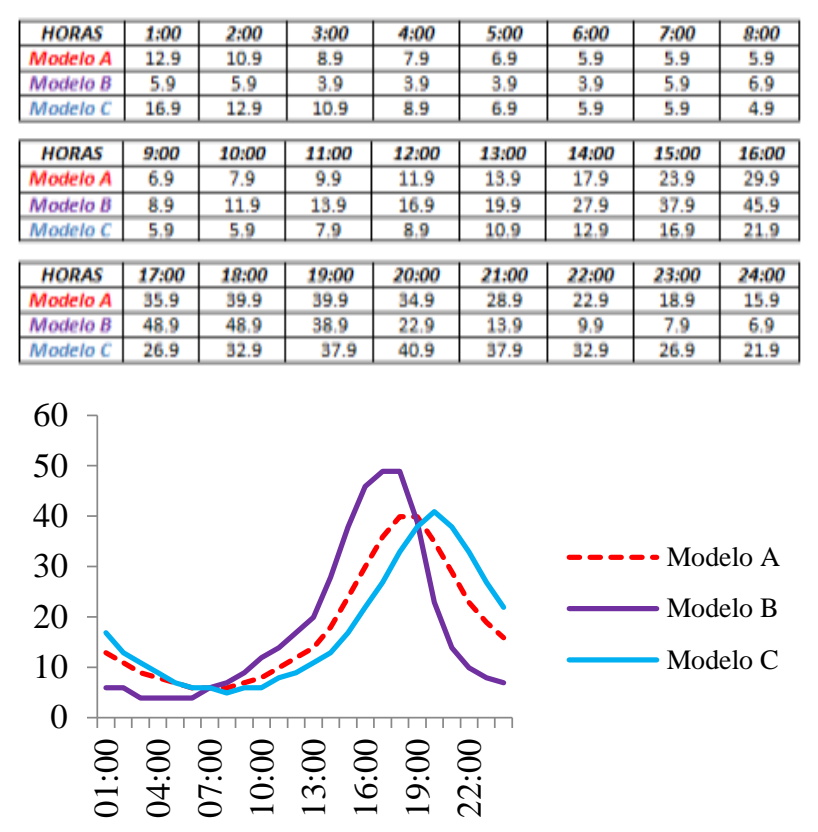

Gráfico 2 Valores CLTD corregidos para las paredes Oeste, de los tres casos, para las $24 \mathrm{hrs}$.

Fuente: Elaboración Propia

El gráfico 3, muestra las demandas de enfriamiento para los tres casos de estudio. Se muestra que sin aislante térmico la demanda de enfriamiento es mayor y para el caso donde se instala aislante térmico disminuye la carga de enfriamiento.

\begin{tabular}{|c|c|c|c|c|c|c|}
\hline HORA & $15: 00$ & $16: 00$ & $17: 00$ & $18: 00$ & $19: 00$ & TOTAL \\
\hline Modelo A & 383.7162 & 395.3276 & 403.8096 & 390.1503 & 365.1225 & 1938.126 \\
\hline Modelo B & 316.4537 & 322.9812 & 311.5958 & 292.5631 & 241.7841 & 1485.377 \\
\hline Modelo C & 107.3477 & 113.3085 & 115.1006 & 115.0942 & 111.3377 & 562.1887 \\
\hline 500 \\
400
\end{tabular}

Gráfico 3 Demanda de enfriamiento para los tres casos de estudio (Watts)

Fuente: Elaboración Propia

La disminución de la demanda de enfriamiento del modelo con aislamiento y aquellos que no están aislados se presenta en la tabla 2, para los tres modelos en porcentaje. 


\begin{tabular}{|l|c|c|c|c|c|c|r|}
\hline Hora & $15: 00$ & $16: 00$ & $17: 00$ & $18: 00$ & $19: 00$ & Promedio \\
\hline $\begin{array}{l}\text { Modelo } \\
\text { A-B }\end{array}$ & 17.52 & 18.30 & 22.83 & 25.01 & 33.78 & 23.36 \\
\hline $\begin{array}{l}\text { Modelo } \\
\text { B-C }\end{array}$ & 66.07 & 64.91 & 63.06 & 60.66 & 53.95 & 62.15 \\
\hline $\begin{array}{l}\text { Modelo } \\
\text { A-C }\end{array}$ & 72.02 & 71.33 & 71.49 & 70.50 & 69.50 & 70.99 \\
\hline
\end{tabular}

Tabla 2 Reducción de la demanda de enfriamiento, con respecto a los tres modelos en $(\%)$

Fuente: Elaboración Propia

En la tabla 2, se muestra un panorama más claro de la comparación de la demanda de enfriamiento para los casos sin aislante térmico y para el caso donde se aplica el aislante. Los valores que se muestran son en porcentajes.

\section{Determinación del consumo de energía de enfriamiento}

Para la determinación del consumo de energía en los tres modelos objeto de estudio para el enfriamiento, se utilizaron wattimetros de $120 \mathrm{~V}$, $60 \mathrm{~Hz}, 1 \mathrm{~F}, 2 \mathrm{H}$ y 9 termómetros con escala de 20 a $50{ }^{\circ} \mathrm{C}$.

1. La temperatura interior fue considerada de $24^{\circ} \mathrm{C}$ de bulbo seco y $50 \%$ de humedad relativa en los tres casos.

2. Las condiciones de temperatura y humedad, fueron tomadas de la estación climatológica perteneciente a la Facultad de ingeniería Ambiental de la Universidad Veracruzana en Poza Rica de Hgo. Veracruz.

3. Se llevaron a cabo mediciones por 28 días en cada uno de los meses de abril, mayo, junio, julio y agosto del 2015.

\section{Consumo de energía para enfriamiento (Resultados)}

Los cálculos de consumo de energía para enfriamiento consistieron en determinar las ganancias térmicas totales a través de la estructura de los modelos a escala A, B y C. Obteniéndose como resultado, los requerimientos energéticos para cada uno de los modelos. El coeficiente de operación de los equipos de aire acondicionado fue considerado de 2.5 .

La tabla 3, muestra la energía de refrigeración en Kw-hr térmico y $\mathrm{Kw}-\mathrm{hr}$ eléctricos para los modelos A, B y C.

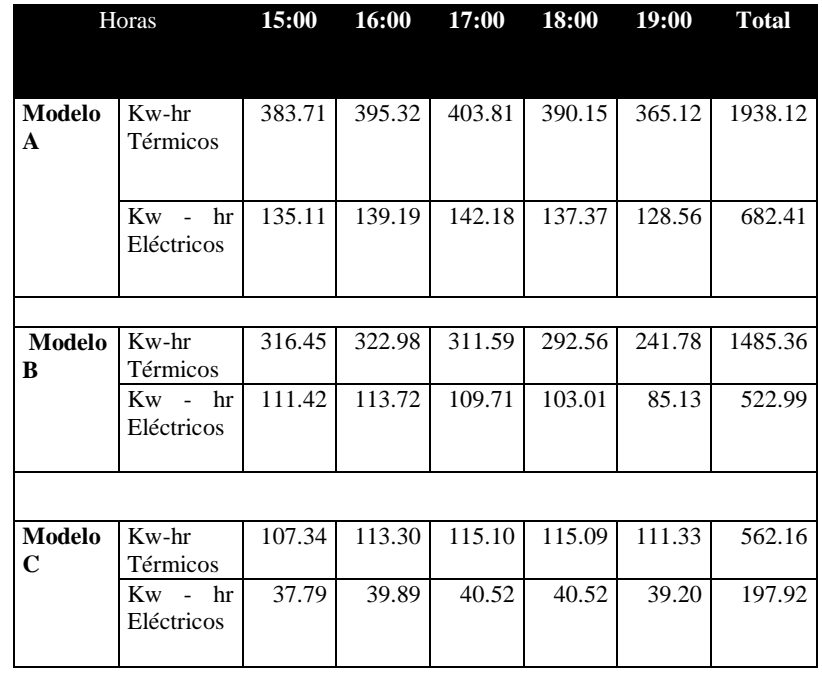

Tabla 3 Energía de refrigeración (Kw - hr Térmicos) y (Kw - hr eléctricos) para los modelos A, B y C.

Fuente: Elaboración Propia

La tabla 4, muestra la energía de refrigeración en $\mathrm{Kw}-\mathrm{hr}$ térmico y $\mathrm{Kw}-\mathrm{hr}$ eléctricos medidos, para los modelos A, B y C.

\begin{tabular}{|c|c|c|c|c|c|c|c|}
\hline & oras & $15: 00$ & 16:00 & $17: 00$ & $18: 00$ & $19: 00$ & Total \\
\hline $\begin{array}{l}\text { Modelo } \\
\text { A }\end{array}$ & $\begin{array}{l}\text { Kw-hr } \\
\text { Térmicos }\end{array}$ & 383.71 & 395.32 & 403.80 & 390.15 & 365.12 & 1938.1 \\
\hline & \begin{tabular}{|l|l|}
$\mathrm{Kw}$ & $-\mathrm{hr}$ \\
Eléctricos
\end{tabular} & 141.02 & 145.40 & 148.53 & 143.50 & 134.30 & 712.86 \\
\hline $\begin{array}{l}\text { Modelo } \\
\text { B }\end{array}$ & $\begin{array}{l}\text { Kw-hr } \\
\text { Térmicos }\end{array}$ & 316.45 & 322.98 & 311.59 & 292.56 & 241.78 & 1485.36 \\
\hline & $\begin{array}{l}\mathrm{Kw}-\mathrm{hr} \\
\text { Eléctricos }\end{array}$ & 118.35 & 120.80 & 116.53 & 109.41 & 90.42 & 555.52 \\
\hline $\begin{array}{l}\text { Modelo } \\
\text { C }\end{array}$ & $\begin{array}{l}\text { Kw-hr } \\
\text { Térmicos }\end{array}$ & 107.34 & 113.30 & 115.10 & 115.09 & 111.33 & 562.16 \\
\hline & $\begin{array}{l}\mathrm{Kw}-\mathrm{hr} \\
\text { Eléctricos }\end{array}$ & 39.68 & 41.88 & 42.54 & 42.54 & 40.95 & 207.81 \\
\hline
\end{tabular}

Tabla 4 Energía de refrigeración (Kw - hr Térmicos) y ( $\mathrm{Kw}$ - hr eléctricos medidos), para los modelos A, B y C. Fuente: Elaboración Propia

\begin{tabular}{|c|c|c|c|c|c|c|}
\hline Modelo & 15:00 & 16:00 & $17: 00$ & 18:00 & 19:00 & $\begin{array}{c}\% \text { de } \\
\text { ahorro }\end{array}$ \\
\hline$A \operatorname{con} B$ & 16.07 & 16.91 & 21.54 & 23.75 & 32.67 & $22.07 \%$ \\
\hline Modelo & $15: 00$ & $16: 00$ & 17:00 & 18:00 & 19:00 & $\begin{array}{ll}\% & \mathrm{de} \\
\text { ahorro } & \end{array}$ \\
\hline $\mathrm{B} \operatorname{con} \mathrm{C}$ & 63.94 & 62.71 & 60.73 & 58.18 & 51.05 & $59.76 \%$ \\
\hline Modelo & 15:00 & 16:00 & 17:00 & 18:00 & 19:00 & $\begin{array}{ll}\% & \mathrm{de} \\
\text { ahorro } & \\
\end{array}$ \\
\hline $\mathrm{A} \operatorname{con} \mathrm{C}$ & 69.74 & 69.02 & 69.19 & 68.11 & 67.04 & $68.64 \%$ \\
\hline
\end{tabular}

Tabla 5 Reducción de la energía eléctrica medida para los modelos A, B y C en (\%)

Fuente: Elaboración Propia

Como se puede apreciar en las tablas 4 y 5 existe un ahorro considerable de energía eléctrica cuando es utilizado aislante térmico en muros y losa en los modelos. 


\section{Anexos}

- $\quad$ ASHRAE (1979, 1992). "Cooling and Heating Load Calculation Manual."

- $\quad$ ASHRAE (1967, 1972, 1985, 1989). "ASHRAE Handbook of Fundamentals". Atlanta, American Society of Heating Refrigerating and Air-Conditioning Engineers, Inc.

- Propiedades térmicas, y números de Código de las capas utilizadas en la descripción de paredes y techos

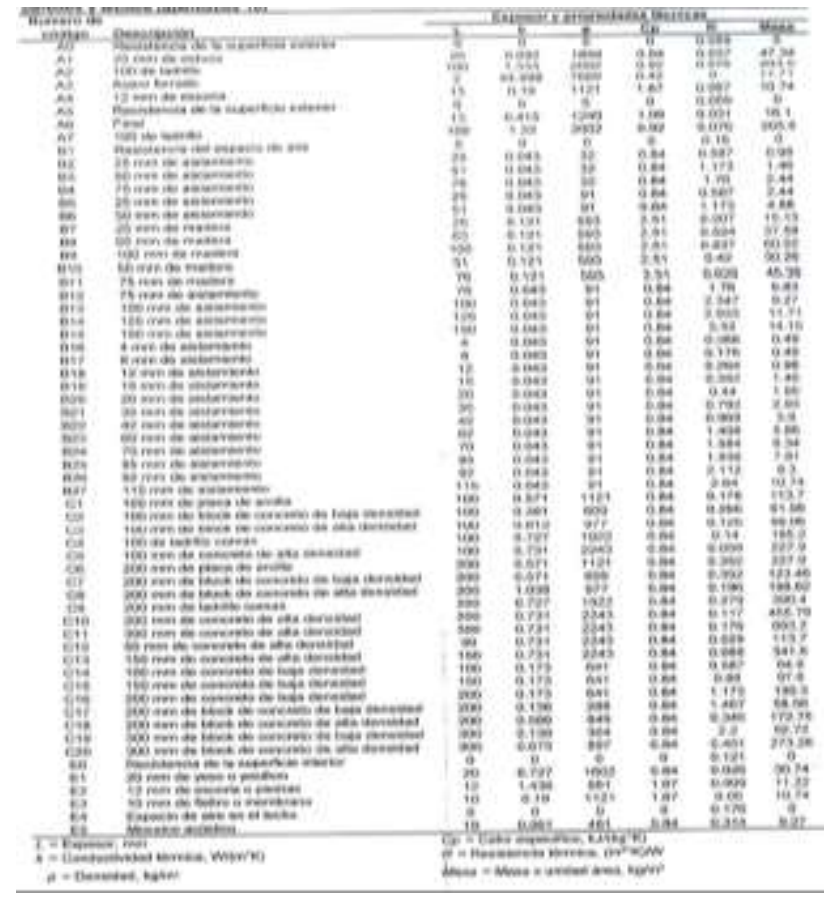

- $\quad$ Numeros de grupo de techo

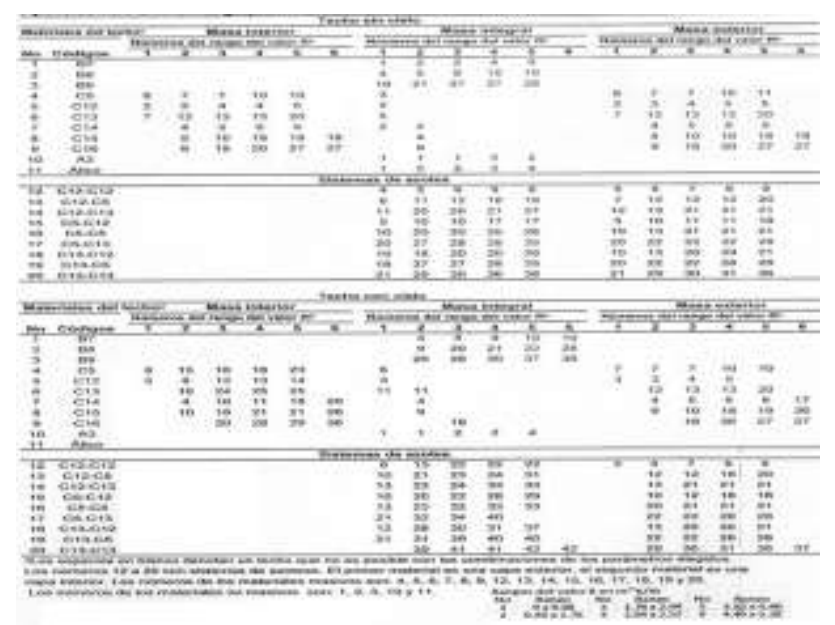

- Diferentes temperaturas de la carga de enfriamiento de Julio para le cálculo de la carga de enfriamiento de los techos planos a $40^{\circ}$ latitud norte

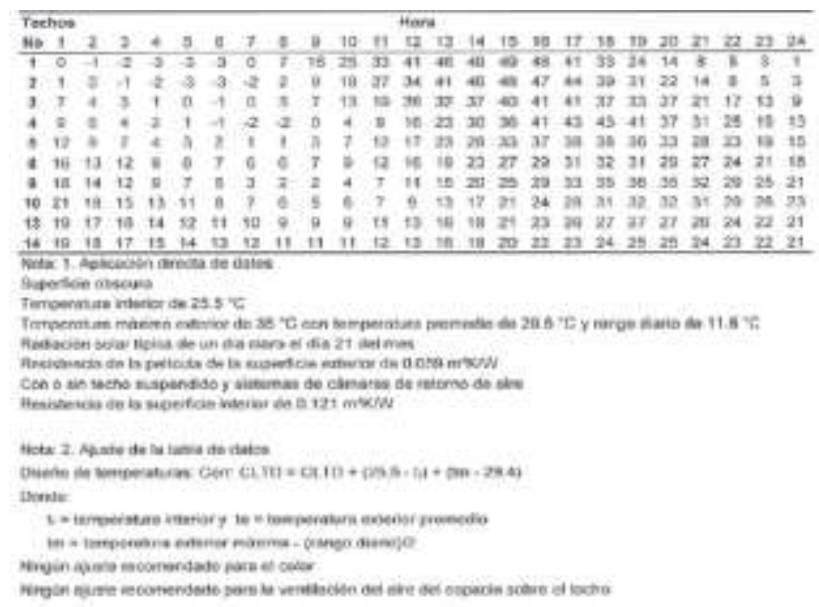

\section{Conclusiones}

Después de desarrollar este trabajo, puede concluirse, que la utilización del método CLTD/SCL/CLF, para el diseño en sistema de aire acondicionado conduce un retraso en la hora pico, reduciendo la carga de enfriamiento, y que la utilización de aislantes térmicos en muros y losas conduce a una disminución en el flujo térmico.

Tomando como base los resultados obtenidos, en este trabajo se observa lo siguiente:

Al utilizar el modelo tipo C, el ahorro de flujo térmico que se obtiene con respecto al tipo A, es del $68.64 \%$, lo cual representa un ahorro considerable en el consumo de energía por lo que se justifica el uso de estos materiales en la construcción de edificios desde el punto de vista energético.

\section{Referencias}

Falconer, D.R., E.F. Sowell, J. D. Spitler, and B. Todorovich. (1993). Electronic tables for the ASHRAE transactions 99

Harris, S. M., \& McQuiston, F.C. (1988). A study to categorize walls and roofs on the basis of thermal response, "ASHRAE Transactions", $94(2), 688-715$.

Lindsey, Kirk (1991). Revision of the CLTD/CLF Cooling Load Calculation Method, M.S. Creative Component, Oklahoma State University.

Machler, M. A., \& Iqbal M. (1985). A modification of the ASHRAE clear sky irradiation model, ASHRAE Transactions, 91(IA), $106-115$.

CASADOS-LÓPEZ, Edzel Jair, CASADOS-SÁNCHEZ, Alvaro, CRUZ-VICENCIO, Raúl y HORST-SÁNCHEZ, Alvaro. Alternativa energética sustentable mediante la utilización de aislantes térmicos de diferentes materiales en edificaciones con sistemas de aire acondicionado. Revista de Energías Renovables. 2020 
McQuiston, F. C., \& Parker, J.D. (1988). Heating, Ventilating, and Air-Conditioning Analysis and Design, John Wiley \& Sons, New York.

Mitalas, G. P., \& Stephenson, D. G. (1967). Cooling Load Calculations by Thermal Response Factor Method, ASHRAE Transactions, 73(I), III.1.1 - 1.7.

Mitalas, G. P., \& Stephenson, D. G. (1967). Room Thermal Response Factors, ASHRAE Transactions, 73(II), III.2.1 - 2.10.

Mitallas, G. P. (1969). An experimental check on the weighting factor method of calculating room cooling load, ASHRAE Transactions, $75(2), 222-232$.

Mitallas, G. P., \& Stephenson, D. G. (1971). Calculation of heat conduction transfer functions for multi - layer slabs, ASHRAE Transactions, $77(2), 117-126$.

Mitallas, G. P. (1971). Transfer Function Method of calculating cooling loads, heat extraction and space temperature, ASHRAE Journal, 12, $54-56$.

Romine, T. B. (Jr.) (1992). Cooling Load Calculation: Art or Science?, ASHRAE Journal, $34,14-24$

Rudoy, W., \& Duran, F. (1975). Development of an Improved Cooling Load Calculation Method, ASHRAE Transactions, 81(2), 19-69.

Spitler, J. D., McQuiston F.C., \& Lindsey K. (1993). The CLTD/SCL/CLF Cooling Load Calculation Method, ASHRAE Transactions.

Spitler, J. D., McQuiston F.C., \& Lindsey K. (1993). Development of a Revised Heating and cooling Load Calculation Manual, ASHRAE Transactions.

Stephenson, D. G. (1968). Calculation of cooling load by digital computer, ASRAE Journal, 4, 41 -43 .

Sowell, E. F., \& Chiles, D. C. (1984a). Characteization of zone dynamic response for CLF/CLTD tables, ASHRAE Transactions, 91(2A), $162-178$.
Sowell, E. F., \& Chiles, D. C. (1984b). Zone descriptions and response characterizations for CLF/CLTD calculations, ASHRAE Transactions, 91(2A), $179-200$.

Sowell, E. F., \& Chiles, D. C. (1984c). A counter - intuitive effect of mass on zone cooling load response, ASHRAE Transactions, 91(2A), 201 208.

Sowell, E. F. (1988a). Load calculations for 200, 640 zones, ASHRAE Transactions, 94(2), 716 736.

Sowell, E. F. (1988b). Cross-check and modification of the DOE-2 program for calculation of zone weighting factors, ASHRAE Transactions, 94(2), $737-753$.

Sowell, E. F. (1988c). Classifiaction of 200, 640 parametric zones for cooling load calculations. ASHRAE Transactions, 94(2), $754-777$.

Threlkeld, J. L. (1963). Solar Irradiation of Surfaces on Clear Days, ASHRAE Transactions, $69,24-36$.

Threlkeld, J. L. \& Jordan R. C. (1959). Direct Solar Radiation available on Clear Days, ASHRAE Transactions, 64, $45-68$.

Todorovic B. (1982). Cooling Loads from Solar Radiation through Partially Shaded Windows, taking Heat Storage Effect into Account, ASHRAE Transactions, 88(2), $924-937$. 